\title{
Zur Entwicklung der Schweizer Spitäler und Heilanstalten im Vergleich mit Nachbarländern
}

\section{Editorial}

Von Huldrych M. Koelbing

Der Anregung ihres Sekretärs, Dr.med.Alfred Gubser, folgend, hat die SGGMN im Jahre 1980 ihre Mitglieder eingeladen, für die jährliche Tagung der Gesellschaft Beiträge zur schweizerischen Spitalgeschichte vorzubereiten. Die Idee fiel auf fruchtbaren Boden. Am 17. Oktober 1980 konnten wir im Rahmen unserer Winterthurer Zusammenkunft ein eigentliches Symposium durchführen, an dem ein bunter Strauß von Referaten zum Thema vorgetragen und diskutiert wurde - jedes auf eine andere Form der Institution, ein anderes Anliegen der Betreuung gerichtet. Nicht eine umfassende, systematische Übersicht der schweizerischen Krankenhausgeschichte wurde geboten oder auch nur angestrebt - hierfür wäre ein dickes Buch nötig -, wohl aber sollte die Vielfalt der Bedürfnisse, der Probleme und der Lösungen deutlich gemacht werden. Dies ist gelungen, wie das nun vorliegende Heft zeigt. Es vereinigt die in Winterthur gehaltenen Vorträge mit zwei weiteren Beiträgen zu der vielseitigen Thematik (Bischoff und Speiser; Hörger). Das Ganze als Sonderheft unserer Zeitschrift zu publizieren war nur möglich, weil die Jubiläumsstiftung der Schweizerischen Mobiliar-Versicherungsgesellschaft den Löwenanteil der Druckkosten übernahm; der Präsidentin des Stiftungsrates, Frau Prof.Dr.Hedi Fritz-Niggli in Zürich, und seinem Sekretär, Herrn Direktor Dr. Otto Saxer in Bern, gilt deshalb unser besonderer Dank.

Als Gast der Dr. Markus-Guggenheim-Schnurr-Stiftung konnten wir den bekannten deutschen Krankenhaushistoriker Axel Hinrich Murken zu einem Hauptvortrag einladen. Seine Ausführungen über das deutsche Krankenhauswesen 1780-1930 im Vergleich mit der Schweiz stellen unser Thema in einen weiteren räumlichen Rahmen. Mit wechselseitigen Einflüs- 
sen war von vornherein zu rechnen; schweizerische Klinikdirektoren und Spitalbaukommissionen haben sich nicht selten persönlich im Ausland umgesehen, um ihren Geist für die trockenen Planungsaufgaben befruchten zu lassen. Mit Befriedigung läßt sich der Schweizer jedoch nun von Murken zeigen, daß auch unsere Schöpfungen Vorbild für deutsche Spitalplaner sein konnten. Während die wissenschaftliche Medizin in der Schweiz, vor allem an den Universitäten von Basel und Zürich, bis zum Ersten Weltkrieg in hohem Maße von deutschen Professoren gepflegt und gelehrt wurde, zeigten also im Spitalwesen die Schweizer eine größere Selbständigkeit.

Die Entwicklung des Krankenhauses in seinen verschiedenen Spielarten wurde und wird eben nicht nur durch die Entwicklung der Medizin bestimmt. Unabhängig von den größeren oder kleineren Möglichkeiten ärztlicher Behandlung dient es dringenden persönlichen und sozialen Bedürfnissen. Die ersten Spitalgründungen entstanden in der Spätantike im östlichen Mittelmeerraum als Ausdruck christlicher Nächstenliebe. Die BenediktinerRegel, die im Kloster St.Gallen zu den Anfängen institutionalisierter Krankenpflege führte, war in Italien geschaffen worden. Einmal mehr erkennen wir uns als Nutznießer und Erben mediterraner Zivilisation. Später wurden die Italiener für die Pestbekämpfung wegweisend. Pestspitäler und Siechenhäuser für Aussätzige lassen eine andere Funktion des Krankenhauses hervortreten: es hat auch Leute aufzunehmen, die ihren Mitmenschen gefährlich erscheinen oder zur Last fallen. Menschenfreundliche Zuwendung und utilitaristische Absonderung - beides hat zur Entwicklung der Krankenhäuser beigetragen. Das zweite Moment wird heute im Blick auf die psychiatrische Klinik gerne tendenziös überbetont; Walsers Artikel in diesem Heft könnte zu einer ausgewogeneren Beurteilung beitragen! Ein weiterer, nachgerade kostspieliger Impuls zur Krankenhausentwicklung kommt aus dem Bedürfnis nach medizinischer Forschung; Anfänge dieser Krankenhaus-Funktion im Großbritannien des 18. Jahrhunderts sind bisher kaum beachtet worden und rechtfertigen den Einschluß einer entsprechenden Arbeit in unser helvetisches Heft.

Wieweit ist ein anderer Zug unseres Krankenhauswesens eine schweizerische Besonderheit, nämlich der große Anteil gemeinnütziger Privat-Initiative an der Entwicklung bis in die Gegenwart hinein? Die Basler Augenklinik wie das Zürcher Kinderspital und einige andere Universitätskliniken haben heute noch die Rechtsform privater Stiftungen, wenn sie auch schon längst nicht mehr ohne Unterstützung durch die öffentliche Hand auskommen können. 
Einen Mangel unseres Symposions bedaure ich besonders: die Suisse romande ist nicht berücksichtigt. Der Grund hiefür ist banal: wir haben keinen Autor gefunden. Vielleicht regt jedoch gerade unsere Publikation zu weiteren Studien an. Fundierte, kurze Beiträge zu dem weiten und wichtigen Thema sind willkommen!

Prof. Dr.med. Huldrych M. Koelbing

Medizinhistorisches Institut der Universität Zürich

Rämistraße 71

CH-8006 Zürich 